\title{
A Reduced-Order Extrapolation Spectral-Finite Difference Scheme Based on the POD Method for 2D Second-Order Hyperbolic Equations*
}

\author{
Zhendong $\mathbf{L u o}^{a}$ and Shiju $\mathrm{Jin}^{b}$ \\ ${ }^{a}$ School of Mathematics and Physics, North China Electric Power University \\ 102206 Beijing, China \\ ${ }^{b}$ School of Control and Computer Engineering, North China Electric Power \\ University \\ 102206 Beijing, China \\ E-mail(corresp.): zhdluo@ncepu.edu.cn \\ E-mail: jinshiju258@163.com
}

Received December 7, 2016; revised May 20, 2017; published online September 15, 2017

\begin{abstract}
In this study, a reduced-order extrapolation spectral-finite difference (ROESFD) scheme based on the proper orthogonal decomposition (POD) method is set up for the two-dimensional (2D) second-order hyperbolic equations. First, the classical spectral-finite difference (CSFD) method for the 2D second-order hyperbolic equations and its stability, convergence, and flaw are introduced. Then, a new ROESFD scheme that has very few degrees of freedom but holds sufficiently high accuracy is set up by the POD method and its implementation is offered. Finally, three numerical examples are offered to explain the validity of the theoretical conclusion. This implies that the ROESFD scheme is viable and efficient for searching the numerical solutions of the $2 \mathrm{D}$ second-order hyperbolic equations.
\end{abstract}

Keywords: reduced-order extrapolation spectral-finite difference scheme, proper orthogonal decomposition, second-order hyperbolic equations, classical spectral-finite difference method, error estimates.

AMS Subject Classification: 65N12; 65M15; 65N30.

\section{Introduction}

For convenience and without loss of universality, let $\Omega=(a, b) \times(c, d) \subset$ $R^{2}$ and consider the following two-dimensional (2D) second-order hyperbolic

* This work was supported by National Science Foundation of China (11671106). 
equations:

$$
\begin{cases}u_{t t}-\Delta u=f(x, y, t), & (x, y, t) \in \Omega \times[0, T] \\ u(x, y, t)=0, & (x, y, t) \in \partial \Omega \times[0, T] \\ u(x, y, 0)=f_{1}(x, y), u_{t}(x, y, 0)=f_{2}(x, y), & (x, y) \in \Omega\end{cases}
$$

where $u_{t t}=\frac{\partial^{2} u}{\partial t^{2}}, \Delta u=\frac{\partial^{2} u}{\partial x^{2}}+\frac{\partial^{2} u}{\partial y^{2}}, f(x, y, t), f_{1}(x, y)$ and $f_{2}(x, y)$ are the given functions, $T$ is the total time.

In recent years, there have been many numerical solution researches for the hyperbolic equations (1.1), also called the wave equations, because they hold very important physical background and have many applications in fluid mechanics problems such as aviation, weather, ocean and so on $[18,24,28$, 30]. As we all know, the numerical methods for PDEs have mainly finite difference $(\mathrm{FD})$ scheme, finite element (FE) method, finite volume element (FVE) method, and spectral method. The FD, FE, and FVE methods build on local argument, while the spectral method is fit to global cases. By comparison, the spectral method can provide superior accuracy (see $[5,25]$ ). The spectral method is one of the weighted residual numerical methods for PDEs, which can be classified as Galerkin type (spectral method) and collocation type (pseudospectral method) (see $[5,10,25,29])$. In this study, we will pay our attention to Galerkin type, also called spectral-Galerkin method.

Although it is viable in theory to find the numerical solution of PDEs by dint of the classical spectral-finite difference (CSFD) method (see $[5,10,25,29]$ ), it still includes many unknowns (degrees of freedom) for a large engineering problem. Due to the truncated error accumulation, the CSFD numerical solutions are frequently appear not to converge in the calculation process. Therefore, for the real engineering problems, under guaranteeing numerical solutions with sufficiently high accuracy, it is very significant how to reduce the degrees of freedom in the CSFD scheme.

The proper orthogonal decomposition (POD) method (see [26]) is a resultful way to reduce the freedom degrees for numerical computation and alleviate the truncation error in calculation process. The reduce-order methods based on the POD technique can not only save calculation time and improve computation efficiency, but also guarantee the accuracy for numerical solutions. In recent years, the POD method has been used to reduce the orders in the some numerical computations (see $[2,3,4,6,7,22,23]$ ). Especially, the reduced-order numerical methods based on the POD method, such as the reduced-order FD schemes, the reduced-order FE methods, reduced-order FVE methods, and the reduced-order spectral methods, have been intensively applied in the numerical calculation for PDEs (see $[8,9,14,20,21,27]$ ), which can be regard as the historical breakthrough for solving PDEs. But, most reduced-order methods as stated above were set up by the POD basis obtained from the classical numerical solutions in the entire time span, before recalculating the approximate solutions on the same time span, which are the meaningless repeating calculations. Since 2013, several reduced-order extrapolating algorithms based on the POD method for PDEs have been proposed successively in Luo's papers $[1,11,12,13,15,16,17,19]$ to escape the meaningless repeating calculation 
in the reduce order procedure as mentioned above.

However, based on what we know, there is yet not any report about the reduced-order extrapolating spectral-FD (ROESFD) scheme based on the POD method, especially, no article about the ROESFD scheme for the 2D secondorder hyperbolic equations has been published. Therefore, in this article, we intend to set up the ROESFD scheme based on the POD method and the spectral-Galerkin method for the 2D second-order hyperbolic equations.

Though reference [1] is the first paper that combines the spectral method with the reduced-order extrapolating scheme based on the POD method for the parabolic equation, it is different from our current method. The major difference consists in that reference [1] adopts the spectral-collocation method whereas our current approach uses spectral-Galerkin method so that both techniques are thoroughly different. Moreover, although reference [1] presented a numerical example to explain its feasibility, there are not visual and vivid figures to contrast the reduced-order spectral approach with the classical spectral scheme. And, reference [1] studied the parabolic equation that only includes the first-order partial derivative about time. In this work, we will set up the ROESFD scheme based on the POD method for the 2D second-order hyperbolic equations with the second-order partial derivative about time, provide some numerical examples, and display the numerical results of the POD-based ROESFD solutions in graphs.

The new ROESFD scheme based on the POD method only uses the CSFD numerical solutions on a small period of time to compose the POD basis. Therefore, it not only includes the merit of the CSFD method with superior accuracy, but also has not repeated computation like references $[1,11,12,13,15,16,17,19]$. Thus, the ROESFD scheme based on the POD method is a significant development and improvement over the former reduced-order methods based on the POD method as mentioned above.

The subsequent contents of this article is organized as follows. In Section 2, we build the CSFD scheme for the 2D hyperbolic equations and offer its convergence and stability analysis. In Section 3, by formulating POD basis, we develop the ROESFD scheme based on the POD method that contains very fewer degrees of freedom but has efficiently precision and offer the error analysis of ROESFD solutions and the implementations for the ROESFD scheme. In Section 4, we supply three familiar numerical examples to validate the feasibility and availability of the ROESFD scheme. In last Section 5, some conclusions and discussion are supplied.

\section{The CSFD scheme for the 2D second-order hyperbolic equations}

\subsection{The spectral-Galerkin method}

When solving the time-dependent PDEs numerically by spectral-FD method, we use the FD in time and the spectral differentiation in space. The main idea of the CSFD algorithm is to look for an approximate solution that satisfies the desirable accuracy at a set of nodes. In this paper, we shall use the most 
familiar example of a set of points as the space nodes, the so-called Chebyshevtype points.

It is well know that the fundamental of the weighted residual method is to get an approximation solution for $u$ by a sum

$$
u(x) \approx u_{N}(x)=\sum_{k=0}^{N} \widehat{u}_{k} \phi_{k}(x),
$$

where $\phi_{k}$ 's are the basis functions and the expansion coefficients $\widehat{u}_{k}$ 's are undetermined.

For convenience, in the following, we set $a=c=-1$ and $b=d=1$ (in fact, using transforms $x^{\prime}=2(x-a) /(b-a)-1$ and $y^{\prime}=2(y-c) /(d-c)-1$, we can ensure $[a, b] \leftrightarrow[-1,1]$ and $[c, d] \leftrightarrow[-1,1]$, respectively). We use the Galerkin type, i.e., spectral-Galerkin method, and chose the Chebyshev polynomials as basis functions. By taking these polynomials in equation (2.1), we achieve the approximation solutions of $u$ as follows:

$$
u(x) \approx u_{N}(x)=\sum_{k=0}^{N} \widehat{u}_{k} T_{k}(x),
$$

where $T_{k}$ 's are the Chebyshev polynomials, which are orthogonal with respect to the Chebyshev weight function $\rho(x)=\frac{1}{\sqrt{1-x^{2}}}$ over $[-1,1]$, namely,

$$
\int_{-1}^{1} T_{n}(x) T_{m}(x) \rho(x) d x=\gamma_{n} \delta_{m, n},
$$

where $\gamma_{n}=\left\|T_{n}(x)\right\|_{\rho}^{2}$. The three-term recurrence relation for the Chebyshev polynomials is as follows:

$$
T_{n+1}(x)=2 x T_{n}(x)-T_{n-1}(x), \quad n \geqslant 1,
$$

where $T_{0}(x)=1$ and $T_{1}(x)=x$. Set the Chebyshev-Gauss-Lobatto (CGL) points $\left\{x_{j}\right\}_{j=0}^{N}$ as the space nodes for the spectral method,

$$
\theta_{j}=j \pi / N, \quad x_{j}=\cos \left(\theta_{j}\right)=\cos (j \pi / N), \quad 0 \leqslant j \leqslant N .
$$

We find that

$$
T_{n}(x)=\cos n \theta, \quad \theta=\arccos x, \quad n \geqslant 0 .
$$

This distinct expression enables us to derive many important properties. One explicit consequence is the recurrence relation:

$$
2 T_{n}(x)=\frac{1}{n+1} T_{n+1}^{\prime}(x)-\frac{1}{n-1} T_{n-1}^{\prime}(x), \quad n \geqslant 2 .
$$

Another can also derive from the above formula that

$$
T_{n}^{\prime}(x)=2 n \sum_{k=0, k+n \text { odd }}^{n-1} \frac{1}{c_{k}} T_{k}(x), \quad 1 \leqslant n \leqslant N
$$

and

$$
T_{n}^{\prime \prime}(x)=\sum_{k=0, k+n \text { even }}^{n-2} \frac{1}{c_{k}} n\left(n^{2}-k^{2}\right) T_{k}(x), \quad 2 \leqslant n \leqslant N,
$$

where $c_{0}=c_{N}=2$ and $c_{k}=1(k=1,2, \ldots, N-1)$. 


\subsection{Establishment of the CSFD scheme}

Let $\Delta t$ be the time step, $\left\{x_{m}\right\}$ and $\left\{y_{l}\right\}$ be the space nodes in $x$ and $y$ directions, respectively, with

$$
x_{m}=\cos \frac{m \pi}{N}, \quad y_{l}=\cos \frac{l \pi}{N}, \quad 0 \leqslant m, l \leqslant N .
$$

Given $u \in C[-1,1], \quad u_{m, l}^{k}(0 \leqslant m, l \leqslant N)$ denote the Chebyshev approximate values of function $u$ at points $\left(x_{m}, y_{l}, k \Delta t\right)$. Thanks to the forward discrete Chebyshev transform:

$$
\widehat{u}_{n}=\frac{2}{c_{n} N} \sum_{j=0}^{N} \frac{1}{c_{j}} u\left(x_{j}\right) \cos \frac{n j \pi}{N}, \quad 0 \leqslant n \leqslant N
$$

and the backward discrete Chebyshev transform:

$$
u\left(x_{j}\right)=\sum_{n=0}^{N} \widehat{u}_{n} T_{n}\left(x_{j}\right)=\sum_{n=0}^{N} \widehat{u}_{n} \cos \frac{n j \pi}{N}, \quad 0 \leqslant j \leqslant N,
$$

we have these representations:

$$
u\left(x_{m}, y_{l}, k \Delta t\right) \approx u_{m, l}^{k}=\sum_{p=0}^{N} \sum_{q=0}^{N} \widehat{u}_{p, q}^{k} T_{p}\left(x_{m}\right) T_{q}\left(y_{l}\right), \quad 0 \leqslant m, l \leqslant N,
$$

namely,

$$
\begin{aligned}
& u_{m, l}^{k}=\sum_{n=0}^{N} Y_{n}^{k} T_{n}\left(x_{m}\right), \quad 0 \leqslant m \leqslant N, \\
& Y_{n}^{k}=\frac{2}{c_{n} N} \sum_{m=0}^{N} \frac{1}{c_{m}} u\left(x_{m}, y_{l}, k \Delta t\right) \cos \frac{n m \pi}{N}, \quad 0 \leqslant n \leqslant N
\end{aligned}
$$

and

$$
\begin{aligned}
& u_{m, l}^{k}=\sum_{n=0}^{N} X_{n}^{k} T_{n}\left(y_{l}\right), \quad 0 \leqslant l \leqslant N, \\
& X_{n}^{k}=\frac{2}{c_{n} N} \sum_{l=0}^{N} \frac{1}{c_{m}} u\left(x_{m}, y_{l}, k \Delta t\right) \cos \frac{n l \pi}{N}, \quad 0 \leqslant n \leqslant N,
\end{aligned}
$$

where $x_{m}=\cos \theta_{m}$ and $y_{l}=\cos \theta_{l}$.

The second derivative on the Chebyshev grid is given by

$$
\frac{\partial^{2} u_{m, l}^{k}}{\partial x^{2}}=\sum_{n=0}^{N} Y_{n}^{k} T_{n}^{\prime \prime}\left(x_{m}\right)=\sum_{n=0}^{N} Y_{n}^{k} \sum_{k=0, k+n \text { even }}^{n-2} \frac{1}{c_{k}} n\left(n^{2}-k^{2}\right) T_{k}\left(x_{m}\right) .
$$

In the same way, we come to the conclusion

$$
\frac{\partial^{2} u_{m, l}^{k}}{\partial y^{2}}=\sum_{n=0}^{N} X_{n}^{k} T_{n}^{\prime \prime}\left(y_{m}\right)=\sum_{n=0}^{N} X_{n}^{k} \sum_{k=0, k+n \text { even }}^{n-2} \frac{1}{c_{k}} n\left(n^{2}-k^{2}\right) T_{k}\left(y_{l}\right) .
$$


For the derivative of time, we use the central finite difference scheme, namely,

$$
\frac{\partial^{2} u_{m, l}^{k}}{\partial t^{2}}=\frac{u_{m, l}^{k+1}-2 u_{m, l}^{k}+u_{m, l}^{k-1}}{\Delta t^{2}} .
$$

Put $u_{n}^{k}=u_{m, l}^{k}, f_{n}^{k}=f_{m, l}^{k}=f\left(x_{m}, y_{l}, k \Delta t\right), f_{2_{n}}=f_{2_{m, l}}=f_{2}\left(x_{m}, y_{l}\right)(1 \leqslant$ $n=m+1+l(N+1) \leqslant M=(N+1)(N+1), 0 \leqslant m \leqslant N, 0 \leqslant l \leqslant N, 0 \leqslant k \leqslant K)$. Then, the solutions to PDEs (1.1) at the nodes can be donated by the set $\left\{u_{n}^{k}\right\}_{k=1}^{K}(1 \leqslant n \leqslant M)$. Let $\boldsymbol{u}^{k}=\left(u_{1}^{k}, u_{2}^{k}, \ldots, u_{M}^{k}\right)^{T}, \boldsymbol{F}^{k}=\left(f_{1}^{k}, f_{2}^{k}, \ldots, f_{M}^{k}\right)^{T}$, and $\boldsymbol{F}_{2}=\left(f_{2_{1}}, f_{2_{2}}, \ldots, f_{2_{M}}\right)$. Then, we can set up the following matrix relationship.

$$
\begin{aligned}
& \frac{\partial^{2}}{\partial x^{2}}\left[\begin{array}{c}
u^{k}\left(x_{0}, y_{l}\right) \\
u^{k}\left(x_{1}, y_{l}\right) \\
\vdots \\
u^{k}\left(x_{N}, y_{l}\right)
\end{array}\right]=\left[\begin{array}{cccc}
T_{0}^{\prime \prime}\left(x_{0}\right) & T_{1}^{\prime \prime}\left(x_{0}\right) & \cdots & T_{N}^{\prime \prime}\left(x_{0}\right) \\
T_{0}^{\prime \prime}\left(x_{1}\right) & T_{1}^{\prime \prime}\left(x_{1}\right) & \cdots & T_{N}^{\prime \prime}\left(x_{1}\right) \\
\vdots & \vdots & \ddots & \vdots \\
T_{0}^{\prime \prime}\left(x_{N}\right) & T_{1}^{\prime \prime}\left(x_{N}\right) & \cdots & T_{N}^{\prime \prime}\left(x_{N}\right)
\end{array}\right]\left[\begin{array}{c}
Y_{0}^{k}(l) \\
Y_{1}^{k}(l) \\
\vdots \\
Y_{N}^{k}(l)
\end{array}\right] \\
& \left\{T_{i}^{\prime \prime}\left(x_{j}\right)\right\}_{i, j=0}^{N}=T_{x}^{T} K^{T}, \quad\left[\begin{array}{c}
Y_{0}^{k}(l) \\
Y_{1}^{k}(l) \\
\vdots \\
Y_{N}^{k}(l)
\end{array}\right]=J\left[\begin{array}{c}
u^{k}\left(x_{0}, y_{l}\right) \\
u^{k}\left(x_{1}, y_{l}\right) \\
\vdots \\
u^{N}\left(x_{0}, y_{l}\right)
\end{array}\right], \\
& \frac{\partial^{2}}{\partial y^{2}}\left[\begin{array}{c}
u^{k}\left(x_{m}, y_{0}\right) \\
u^{k}\left(x_{m}, y_{1}\right) \\
\vdots \\
u^{k}\left(x_{m}, y_{N}\right)
\end{array}\right]=\left[\begin{array}{cccc}
T_{0}^{\prime \prime}\left(y_{0}\right) & T_{1}^{\prime \prime}\left(y_{0}\right) & \cdots & T_{N}^{\prime \prime}\left(y_{0}\right) \\
T_{0}^{\prime \prime}\left(y_{1}\right) & T_{1}^{\prime \prime}\left(y_{1}\right) & \cdots & T_{N}^{\prime \prime}\left(y_{1}\right) \\
\vdots & \vdots & \ddots & \vdots \\
T_{0}^{\prime \prime}\left(y_{N}\right) & T_{1}^{\prime \prime}\left(y_{N}\right) & \cdots & T_{N}^{\prime \prime}\left(y_{N}\right)
\end{array}\right]\left[\begin{array}{c}
X_{0}^{k}(m) \\
X_{1}^{k}(m) \\
\vdots \\
X_{N}^{k}(m)
\end{array}\right] \text {, } \\
& \left\{T_{i}^{\prime \prime}\left(y_{j}\right)\right\}_{i, j=0}^{N}=T_{y}^{T} K^{T}, \quad\left[\begin{array}{c}
X_{0}^{k}(l) \\
X_{1}^{k}(l) \\
\vdots \\
X_{N}^{k}(l)
\end{array}\right]=J\left[\begin{array}{c}
u^{k}\left(x_{m}, y_{0}\right) \\
u^{k}\left(x_{m}, y_{1}\right) \\
\vdots \\
u^{N}\left(x_{m}, y_{N}\right)
\end{array}\right],
\end{aligned}
$$

where,

$$
\begin{aligned}
& \boldsymbol{T}_{y}=\boldsymbol{T}_{x} \\
& =\left[\begin{array}{cccc}
\cos \frac{(1-1)(1-1) \pi}{N} & \cos \frac{(1-1)(2-1) \pi}{N} & \cdots & \cos \frac{(1-1)(N+1-1) \pi}{N} \\
\cos \frac{(2-1)(1-1) \pi}{N} & \cos \frac{(2-1)(2-1) \pi}{N} & \cdots & \cos \frac{(2-1)(N+1-1) \pi}{N} \\
\vdots & \vdots & \ddots & \vdots \\
\cos \frac{(N+1-1)(1-1) \pi}{N} & \cos \frac{(N+1-1)(2-1) \pi}{N} & \cdots & \cos \frac{(N+1-1)(N+1-1) \pi}{N}
\end{array}\right],
\end{aligned}
$$




$$
\begin{aligned}
& \boldsymbol{K}=\left[\boldsymbol{K}_{0}, \boldsymbol{K}_{1}, \boldsymbol{K}_{2}, \boldsymbol{K}_{3}, \ldots, \boldsymbol{K}_{N-2}, \boldsymbol{O}, \boldsymbol{O}\right]_{(N+1) \times(N+1)}, \\
& \boldsymbol{K}_{0}=\left(0,0,2^{3} / 2,0,4^{3} / 2, \ldots, 0, N^{3} / 2\right)^{T} \text {, } \\
& \boldsymbol{K}_{1}=\left(0,0,0,3^{3}-3,0, \ldots,(N-1)^{3}-(N-1), 0\right)^{T} \text {, } \\
& \boldsymbol{K}_{2}=\left(0,0,0,0,4^{3}-4 * 2^{2}, \ldots, 0, N^{3}-N * 2^{2}\right)^{T}, \\
& \boldsymbol{K}_{3}=\left(0,0,0,0,0, \ldots,(N-1)^{3}-(N-1) * 3^{2}, 0\right)^{T}, \\
& \boldsymbol{K}_{N-2}=\left(0,0,0,0,0, \ldots, 0, N^{3}-N *(N-2)^{2}\right)^{T}, \\
& \boldsymbol{J}=\frac{2}{N}\left[\boldsymbol{J}_{0}, \boldsymbol{J}_{1}, \boldsymbol{J}_{2}, \boldsymbol{J}_{3}, \ldots, \boldsymbol{J}_{N}\right]_{(N+1) \times(N+1)}, \\
& \boldsymbol{J}_{0}=0.5(0.5,1,1, \ldots, 1,0.5)^{T} \text {, } \\
& \boldsymbol{J}_{1}=\left(0.5, \cos \frac{1 \pi}{N}, \cos \frac{2 \pi}{N}, \ldots, \cos \frac{(N-1) \pi}{N},-0.5\right)^{T}, \\
& \boldsymbol{J}_{2}=\left(0.5, \cos \frac{2 \pi}{N}, \cos \frac{4 \pi}{N}, \ldots, \cos \frac{2(N-1) \pi}{N}, 0.5\right)^{T}, \\
& \boldsymbol{J}_{N-1}=\left(0.5 \cos \frac{0 *(N-1) \pi}{N}, \cos \frac{1 *(N-1) \pi}{N}, \cos \frac{2 *(N-1) \pi}{N},\right. \\
& \left.\ldots, \cos \frac{(N-1) *(N-1) \pi}{N}, 0.5 \cos \frac{N *(N-1) \pi}{N}\right)^{T}, \\
& \boldsymbol{J}_{N}=0.5\left(0.5 \cos \frac{0 * N \pi}{N}, \cos \frac{1 * N \pi}{N}, \cos \frac{2 * N \pi}{N}, \ldots\right. \\
& \left.\cos \frac{(N-1) * N \pi}{N}, 0.5 \cos \frac{N * N \pi}{N}\right)^{T} .
\end{aligned}
$$

Further, we can establish the spectral-FD recurrence scheme for PDEs (1.1) as follows

$$
\left\{\begin{array}{l}
\boldsymbol{u}^{k+1}=2 \boldsymbol{u}^{k}-\boldsymbol{u}^{k-1}+\Delta t^{2} \boldsymbol{A} \boldsymbol{u}^{k}+\Delta t^{2} \boldsymbol{B} \boldsymbol{u}^{k}+\Delta t^{2} \boldsymbol{F}^{k}, \\
\quad k=1,2, \ldots, K-1, \\
u_{m, l}^{0}=f_{1}\left(x_{m}, y_{l}\right), \quad m=1,2, \cdots, N-1, \quad l=1,2, \ldots, N-1, \\
u^{1}=0.5 \Delta t^{2} A u^{0}+0.5 \Delta t^{2} B u^{0}+u^{0}+0.5 \Delta t^{2} F^{0}+\Delta t F_{2}, \\
u_{0, l}^{k}=u_{N, l}^{k}=0, \quad l=0,1, \ldots, N, k=0,1, \ldots, K, \\
u_{m, 0}^{k}=u_{m, N}^{k}=0, \quad m=0,1, \ldots, N, k=0,1, \ldots, K,
\end{array}\right.
$$

where $\boldsymbol{B}$ and $\boldsymbol{A}$ are $M \times M$ matrix, which are separately given as follows:

$$
\begin{aligned}
& \boldsymbol{A}=\operatorname{diag}(\boldsymbol{X}), \quad X=\boldsymbol{T}_{x}^{T} \cdot \boldsymbol{K}^{T} \cdot \boldsymbol{J}, \\
& \boldsymbol{B}=[\boldsymbol{D}(i, j)]_{(N+1) \times(N+1)}, \quad \boldsymbol{D}(i, j)=\operatorname{diag}\{Y(i, j), Y(i, j), \ldots, Y(i, j)\}_{N \times N}
\end{aligned}
$$

and $Y(i, j)$ denote the $i$ row and $j$ column elements of matrix $\boldsymbol{Y}=\boldsymbol{T}_{y}^{T} \cdot \boldsymbol{K}^{T} \cdot \boldsymbol{J}$. 


\subsection{Analysis of error and stability condition}

By using the Taylor's expansion, we have

$$
\begin{aligned}
& u_{m, l}^{k+1}=u_{m, l}^{k}+\Delta t \frac{\partial u_{m, l}}{\partial t}+\frac{\Delta t^{2}}{2 !} \frac{\partial^{2} u_{m, l}}{\partial t^{2}}+\frac{\Delta t^{3}}{3 !} \frac{\partial^{3} u_{m, l}}{\partial t^{3}}+O\left(\Delta t^{4}\right), \\
& u_{m, l}^{k-1}=u_{m, l}^{k}-\Delta t \frac{\partial u_{m, l}}{\partial t}+\frac{\Delta t^{2}}{2 !} \frac{\partial^{2} u_{m, l}}{\partial t^{2}}-\frac{\Delta t^{3}}{3 !} \frac{\partial^{3} u_{m, l}}{\partial t^{3}}+O\left(\Delta t^{4}\right) .
\end{aligned}
$$

Therefore, we obtain

$$
\frac{u_{m, l}^{k+1}-2 u_{m, l}^{k}+u_{m, l}^{k-1}}{\Delta t^{2}}-\frac{\partial^{2} u_{m, l}^{k}}{\partial t^{2}}=O\left(\Delta t^{2}\right) .
$$

We introduce an important theorem on the Chebyshev interpolation error estimates (see [25, Chapter III]).

Theorem 1. For any $u \in B_{-1 / 2,-1 / 2}^{q}(I):=\left\{u: \partial_{x}^{l} u \in L_{\omega^{-1 / 2+l,-1 / 2+l}}^{2}(I), 0 \leqslant\right.$ $l \leqslant q\}$ with $m \geqslant 1$, we have

$$
\left\|\partial_{x}^{k}\left(I_{N}^{c}\right) u-u\right\|_{\omega^{k-1 / 2, k-1 / 2}}=O\left(N^{k-q}\right), \quad 0 \leqslant k \leqslant q \leqslant N+1 .
$$

Thus, the error estimates for the CSFD scheme (2.2) are as follows

$$
\left\|u_{m, l}^{k}-u\left(x_{m}, y_{l}, k \Delta t\right)\right\|_{2}=O\left(\Delta t^{2}, N^{2-q}\right), \quad 0 \leqslant 2 \leqslant q \leqslant N+1 .
$$

The stability condition of the CSFD scheme (2.2) for the 2D second-order hyperbolic equations (1.1) is $\Delta t^{2} \leqslant 6.6 N^{-2}$, which has been given at Chapter 10 in the reference [29].

\section{The ROESFD scheme based on the POD method for the $2 \mathrm{D}$ second-order hyperbolic equations}

\subsection{Formulation of the POD basis}

Step 1. Choose snapshots (samples).

Put $u_{n}^{k}=u_{m, l}^{k}(1 \leqslant n=m+1+l(N+1) \leqslant M=(N+1)(N+1), 0 \leqslant$ $m \leqslant N, 0 \leqslant l \leqslant N, 0 \leqslant k \leqslant K)$. Then, we can denote the CSFD solutions to PDEs (1.1) by the set $\left\{u_{n}^{k}\right\}_{k=1}^{K}(1 \leqslant n \leqslant M)$ as mentioned above. By choosing the first $L(L \ll K)$ sequences of solutions $\left\{u_{n}^{k}\right\}_{k=1}^{L}(1 \leqslant n \leqslant M, L \ll M)$ as snapshots, we formulate an $M \times L$ snapshot matrix

$$
\boldsymbol{P}=\left[\begin{array}{cccc}
u_{1}^{1} & u_{1}^{2} & \ldots & u_{1}^{L} \\
u_{2}^{1} & u_{2}^{2} & \ldots & u_{2}^{L} \\
\vdots & \vdots & \ddots & \vdots \\
u_{M}^{1} & u_{M}^{1} & \ldots & u_{M}^{L}
\end{array}\right]
$$

Step 2. Singular value decomposition. 
By singular value decomposition, the snapshot matrix $\boldsymbol{P}$ is denoted by

$$
\boldsymbol{P}=\boldsymbol{U}\left[\begin{array}{cc}
\boldsymbol{S}_{w \times w} & \boldsymbol{O}_{w \times(L-w)} \\
\boldsymbol{O}_{(M-w) \times w} & \boldsymbol{O}_{(M-w) \times(L-w)}
\end{array}\right] \boldsymbol{V}^{T},
$$

where $\boldsymbol{S}_{w \times w}=\operatorname{diag}\left\{\sigma_{1}, \sigma_{2}, \cdots, \sigma_{w}\right\}, w=\operatorname{rank}(P), \sigma_{i}(i=1,2, \ldots, w)$ are the singular values of $\boldsymbol{P}$ sorted in descending order $\sigma_{1} \geqslant \sigma_{2} \geqslant \ldots \geqslant \sigma_{w}>0$, $\boldsymbol{U}=\left(\boldsymbol{\varphi}_{1}, \boldsymbol{\varphi}_{2}, \ldots, \boldsymbol{\varphi}_{M}\right)$ is an $M \times M$ orthogonal matrix, $\boldsymbol{\varphi}_{i}(i=1,2, \ldots, M)$ are the eigenvectors of $\boldsymbol{P} \boldsymbol{P}^{T}$ arranged according to the order of $\sigma_{i}, \boldsymbol{V}=$ $\left(\boldsymbol{\psi}_{1}, \boldsymbol{\psi}_{2}, \ldots, \boldsymbol{\psi}_{L}\right)$ is an $L \times L$ orthogonal matrix, and $\boldsymbol{\psi}_{i}(i=1,2, \ldots, L)$ are the eigenvectors of $\boldsymbol{P}^{T} \boldsymbol{P}$, whose arrangement is the same as $\boldsymbol{\varphi}_{i}$.

Because the number $M$ of the mesh points is much larger than that $L$ of the snapshots extracted, the degree $M$ for matrix $\boldsymbol{P} \boldsymbol{P}^{T}$ is much larger than the that $L$ for matrix $\boldsymbol{P}^{T} \boldsymbol{P}$, but their positive eigenvalues $\lambda_{i}(i=1,2, \ldots, w)$ are uniform and $\lambda_{i}=\sigma_{i}^{2}$. So, first, we solve the eigenvalues $\lambda_{i}(i=1,2, \ldots, w)$ for matrix $\boldsymbol{P}^{T} \boldsymbol{P}$ and the relative eigenvectors $\boldsymbol{\psi}_{i}(i=1,2, \ldots, w)$, and then, by the relationship

$$
\boldsymbol{\varphi}_{i}=\frac{1}{\sigma_{i}} \boldsymbol{P} \boldsymbol{\psi}_{i}, \quad i=1,2, \ldots, w
$$

we achieve the eigenvectors $\boldsymbol{\varphi}_{i}(1 \leqslant i \leqslant w \leqslant L)$ with relating to the nonzero eigenvalues for matrix $\boldsymbol{P} \boldsymbol{P}^{T}$. Thus, we may get matrices $\boldsymbol{U}$ and $\boldsymbol{V}$.

Set $\boldsymbol{\Phi}=\left(\boldsymbol{\varphi}_{1}, \boldsymbol{\varphi}_{2}, \ldots, \boldsymbol{\varphi}_{d}\right)$ and

$$
\boldsymbol{P}_{d}=\boldsymbol{U}\left[\begin{array}{cc}
\boldsymbol{S}_{d \times d} & \boldsymbol{O}_{d \times(L-d)} \\
\boldsymbol{O}_{(M-d) \times d} & \boldsymbol{O}_{(M-d) \times(L-d)}
\end{array}\right] \boldsymbol{V}^{T},
$$

where $\boldsymbol{S}_{d \times d}=\operatorname{diag}\left\{\sigma_{1}, \sigma_{2}, \cdots, \sigma_{d}\right\}(d \leqslant w)$. Thus, we have $\boldsymbol{P}_{d}=\boldsymbol{\Phi} \boldsymbol{\Phi}^{T} \boldsymbol{P}$.

Step 3. Formulate the POD basis.

Define the norm of matrix $\boldsymbol{P}$ as $\|\boldsymbol{P}\|_{2,2}=\sup _{\boldsymbol{u} \in R^{L}} \frac{\|\boldsymbol{P} \boldsymbol{u}\|_{2}}{\|\boldsymbol{u}\|_{2}}$, where $\|\boldsymbol{u}\|_{2}$ is the $l^{2}$ norm for vector $\boldsymbol{u}$. By the relationship between the matrix norm and its spectral radius, we have

$$
\left\|\boldsymbol{P}-\boldsymbol{P}_{d}\right\|_{2,2}=\left\|\boldsymbol{P}-\boldsymbol{\Phi} \boldsymbol{\Phi}^{T} \boldsymbol{P}\right\|_{2,2}=\sqrt{\lambda_{d+1}} .
$$

If we donate $L$ column vectors of $\boldsymbol{P}$ by $\boldsymbol{u}^{k}=\left(u_{1}^{k}, u_{2}^{k}, \ldots, u_{M}^{k}\right)^{T}(k=1,2, \ldots, L)$, we have

$$
\begin{aligned}
& \left\|\boldsymbol{u}^{k}-\boldsymbol{u}_{d}^{k}\right\|_{2}=\left\|\left(\boldsymbol{P}-\boldsymbol{P}_{d}\right) \boldsymbol{\varepsilon}_{k}\right\|_{2} \\
& \quad \leqslant\left\|\boldsymbol{P}-\boldsymbol{P}_{d}\right\|_{2,2}\left\|\varepsilon_{k}\right\|_{2}=\sqrt{\lambda_{d+1}}, \quad k=1,2, \ldots, L,
\end{aligned}
$$

where $\varepsilon_{k}$ 's denote the unit vector with $k$-th component being 1 ,

$$
\boldsymbol{u}_{d}^{k}=\boldsymbol{P}_{d} \varepsilon_{k}=\boldsymbol{\Phi} \boldsymbol{\Phi}^{T} \boldsymbol{P} \varepsilon_{k}=\boldsymbol{\Phi} \boldsymbol{\Phi}^{T} \boldsymbol{u}^{k}=\sum_{j=1}^{d}\left(\boldsymbol{\varphi}_{j}, \boldsymbol{u}_{k}\right) \boldsymbol{\varphi}_{j}
$$

and $\left(\boldsymbol{\varphi}_{j}, \boldsymbol{u}^{k}\right)$ 's are the inner product of $\boldsymbol{\varphi}_{j}$ and $\boldsymbol{u}^{k}(k=1,2, \ldots, L)$. Thus, $\boldsymbol{u}_{d}^{k}(k=1,2, \ldots, L)$ represent the projection of $\boldsymbol{u}^{k}(k=1,2, \ldots, L)$ onto 
$\operatorname{span}\left\{\boldsymbol{\varphi}_{1}, \boldsymbol{\varphi}_{2}, \ldots, \boldsymbol{\varphi}_{d}\right\}$. Apparently, the inequalities (3.1) show that $\boldsymbol{u}_{d}^{k}(k=$ $1,2, \ldots, L)$ are the ideal approximations of $\boldsymbol{u}^{k}(k=1,2, \ldots, L)$, whose errors are no more than $\sqrt{\lambda_{d+1}}$. Therefore, $\boldsymbol{\Phi}$ is exactly an optimal orthogonal POD basis of $\boldsymbol{P}$.

\subsection{Establishment of the ROESFD scheme}

In the Section 3.1, with the POD basis $\boldsymbol{\Phi}$, from the first $L(L \leqslant M)$ solution vectors $\boldsymbol{u}^{k}$ for the CSFD scheme (2.2), we have obtained that the first $L(L \leqslant$ $M)$ optimal approximate solutions $\boldsymbol{u}_{d}^{k}=\boldsymbol{\Phi} \boldsymbol{\Phi}^{T} \boldsymbol{u}^{k}=: \boldsymbol{\Phi} \boldsymbol{\alpha}^{k}(k=1,2, \ldots, L)$, where $\boldsymbol{\alpha}^{k}=\left(\alpha_{1}^{k}, \alpha_{2}^{k}, \ldots, \alpha_{d}^{k}\right)^{T}$ are the vectors associated with $k$. Thus, if the solution vectors $\boldsymbol{u}^{k}$ for the CSFD recurrence scheme (2.2) are approximated by $\boldsymbol{u}_{d}^{k}=\boldsymbol{\Phi} \boldsymbol{\alpha}^{k}(k=1,2, \ldots, L, L+1, \ldots, K)$, i.e., $\boldsymbol{u}^{k}$ replaced with $\boldsymbol{u}_{d}^{k}=$ $\boldsymbol{\Phi} \boldsymbol{\alpha}^{k}(k=1,2, \ldots, L, L+1, \ldots, K)$, we can obtain the ROESFD scheme based on the POD basis as follows:

$$
\left\{\begin{array}{l}
\boldsymbol{u}_{d}^{k}=\boldsymbol{\Phi} \boldsymbol{\Phi}^{T} \boldsymbol{u}^{k}=: \boldsymbol{\Phi} \boldsymbol{\alpha}^{k}, \quad k=1,2, \ldots, L \\
\boldsymbol{\Phi} \boldsymbol{\alpha}^{k+1}=2 \boldsymbol{\Phi} \boldsymbol{\alpha}^{k}-\boldsymbol{\Phi} \boldsymbol{\alpha}^{k-1}+\Delta t^{2} \boldsymbol{A} \boldsymbol{\Phi} \boldsymbol{\alpha}^{k}+\Delta t^{2} \boldsymbol{B} \boldsymbol{\Phi} \boldsymbol{\alpha}^{k}+\Delta t^{2} \boldsymbol{F}^{k} \\
\quad k=L, L+1, \ldots, K-1 .
\end{array}\right.
$$

Due to the matrix $\boldsymbol{\Phi}$ consisting of the orthogonal vectors, the system of equations (3.2) multiplied by $\boldsymbol{\Phi}^{T}$ yields

$$
\left\{\begin{array}{l}
\boldsymbol{\alpha}^{k}=\boldsymbol{\Phi}^{T} \boldsymbol{u}^{k}, \quad k=1,2, \ldots, L \\
\boldsymbol{\alpha}^{k+1}=2 \boldsymbol{\alpha}^{k}-\boldsymbol{\alpha}^{k-1}+\Delta t^{2} \boldsymbol{\Phi}^{T} \boldsymbol{A} \boldsymbol{\Phi} \boldsymbol{\alpha}^{k}+\Delta t^{2} \boldsymbol{\Phi}^{T} \boldsymbol{B} \boldsymbol{\Phi} \boldsymbol{\alpha}^{k}+\Delta t^{2} \boldsymbol{\Phi}^{T} \boldsymbol{F}^{k} \\
\quad k=L, L+1, \ldots, K-1 .
\end{array}\right.
$$

After we have gained $\boldsymbol{\alpha}^{k}(k=1,2, \ldots, L, L+1, \ldots, K)$ from the system of the equations (3.3), we immediately achieve the solution vectors to the ROESFD scheme as follows

$$
\boldsymbol{u}_{d}^{k}=\boldsymbol{\Phi} \boldsymbol{\alpha}^{k}, \quad k=1,2, \ldots, L, L+1, \ldots, K .
$$

Further, we can achieve the ROESFD solution components for the 2D second-order hyperbolic equations (1.1) as follows

$$
u_{d, m, l}^{k}=u_{d n}^{k}, \quad 0 \leqslant m \leqslant N, \quad 0 \leqslant l \leqslant N,
$$

where $m$ and $l$ satisfy $1 \leqslant n=m+1+l(N+1) \leqslant M=(N+1)(N+1)$ and $\boldsymbol{u}_{d}^{k}=\left(u_{d 0}^{k}, u_{d 1}^{k}, \ldots, u_{d n}^{k}, \ldots, u_{d M}^{k}\right)^{T}$ are the solution vectors for the system of the equations (3.3)-(3.4).

Remark 1. It is clearly seen that the CSFD scheme (2.2) contains $M(M=$ $(N+1)(N+1))$ unknowns at each time level, whereas the system of equations (3.3) only contains $d(d<L \ll M)$ unknowns at the same time level. Thus, we can easily realize the advantage of the ROESFD scheme (3.3) based on the POD technique for the $2 \mathrm{D}$ second-order hyperbolic equations. 


\subsection{Error analysis of the ROESFD solutions}

We will give the following discrete Gronwall lemma (see [30]) before analyzing the error.

Lemma 1. If $\left\{a_{n}\right\},\left\{b_{n}\right\}$, and $\left\{c_{n}\right\}$ are three positive sequences, and $\left\{c_{n}\right\}$ is monotone, that satisfy $a_{0}+b_{0} \leqslant c_{0}$ and $a_{n}+b_{n} \leqslant c_{n}+\gamma \sum_{i=0}^{n-1} a_{i}(\gamma>0)$, then $a_{n}+b_{n} \leqslant c_{n} \exp (n \gamma)(n \geqslant 0)$.

Let $\boldsymbol{e}^{k}=\boldsymbol{u}^{k}-\boldsymbol{u}_{d}^{k}$. As $k=1,2, \ldots, L$, from the inequalities (3.1), we have

$$
\left\|\boldsymbol{e}^{k}\right\|_{2}=\left\|\boldsymbol{u}^{k}-\boldsymbol{u}_{d}^{k}\right\|=\left\|\left(\boldsymbol{P}-\boldsymbol{\Phi} \boldsymbol{\Phi}^{T} \boldsymbol{P}\right) \boldsymbol{\varepsilon}_{k}\right\|_{2} \leqslant \sqrt{\lambda_{d+1}} .
$$

As $k=L, L+1, \ldots, K$, by replacing $\boldsymbol{\Phi} \boldsymbol{\alpha}^{k}$ of scheme (3.3) with $\boldsymbol{\Phi} \boldsymbol{\alpha}^{k}=\boldsymbol{u}_{d}^{k}$, we have

$$
\boldsymbol{u}_{d}^{k+1}=2 \boldsymbol{u}_{d}^{k}-\boldsymbol{u}_{d}^{k-1}+\Delta t^{2} \boldsymbol{A} \boldsymbol{u}_{d}^{k}+\Delta t^{2} \boldsymbol{B} \boldsymbol{u}_{d}^{k}+\Delta t^{2} \boldsymbol{F}^{k} .
$$

By subtracting (3.5) from (2.2) and summing from $L$ to $k(k=L, L+$ $1, \ldots, K-1)$, we achieve

$$
\boldsymbol{e}^{k+1}=\boldsymbol{e}^{k}+\boldsymbol{e}^{L}-\boldsymbol{e}^{L-1}+\left[\Delta t^{2} \boldsymbol{A}+\Delta t^{2} \boldsymbol{B}\right] \sum_{j=L}^{k} \boldsymbol{e}_{j}, k=L, L+1, \ldots, K-1 .
$$

Further, we have

$$
\begin{aligned}
& \left\|\boldsymbol{e}^{k+1}\right\|_{2} \leqslant\left\|\boldsymbol{e}^{k}\right\|_{2}+\left\|\boldsymbol{e}^{L}\right\|_{2}+\left\|\boldsymbol{e}^{L-1}\right\|_{2}+\left(\Delta t^{2}\|\boldsymbol{A}\|_{2}+\Delta t^{2}\|\boldsymbol{B}\|_{2}\right) \sum_{j=L}^{k}\left\|\boldsymbol{e}^{j}\right\|_{2} \\
& \leqslant\left\|\boldsymbol{e}^{L}\right\|_{2}+\left\|\boldsymbol{e}^{L-1}\right\|_{2}+\bar{\gamma} \sum_{j=L}^{k}\left\|\boldsymbol{e}^{j}\right\|_{2} \\
& \leqslant 2 \sqrt{\lambda_{d+1}}+\bar{\gamma} \sum_{j=L}^{k}\left\|e^{j}\right\|_{2},
\end{aligned}
$$

where $\bar{\gamma}=1+\Delta t^{2}\|A\|_{2}+\Delta t^{2}\|B\|_{2}$. From Lemma 1, we have

$$
\left\|e^{k+1}\right\|_{2} \leqslant 2 \sqrt{\lambda_{d+1}} \exp [\bar{\gamma}(k-L)], \quad k=L, L+1, \ldots, K-1 .
$$

Because the absolute values of the components of each vector are no more than the vector norm, the errors between the CSFD solutions and the ROESFD solution components have the following estimates:

$$
\left|u_{m, l}^{k}-u_{d, m, l}^{k}\right| \leqslant E(k) \sqrt{\lambda_{d+1}}, \quad 0 \leqslant m \leqslant N, 0 \leqslant l \leqslant N, 0 \leqslant k \leqslant K,
$$

where $E(k)=1(1 \leqslant k \leqslant L)$ and $E(k)=2 \exp \left[\left(1+\Delta t^{2}\|\boldsymbol{A}\|_{2}+\Delta t^{2}\|\boldsymbol{B}\|_{2}\right)(k-L)\right]$ $(L+1 \leqslant k \leqslant K)$.

Moreover, the errors between the exact solution $u$ and the solution ROESFD components $u_{d m, l}^{k}$ for the hyperbolic equations (1.1) have the following estimates

$$
\left|u\left(x_{m}, y_{l}, k \Delta t\right)-u_{d, m, l}^{k}\right|=O\left(E(k) \sqrt{\lambda_{d+1}}, N^{2-q},(\Delta t)^{2}\right),
$$

where $0 \leqslant m \leqslant N, 0 \leqslant l \leqslant N, 0 \leqslant k \leqslant K$. 
Remark 2. The error analysis procedure show that the error factor $\sqrt{\lambda_{d+1}}$ is caused for the reduced-order of the CSFD scheme and $E(k)=2 \exp [(1+$ $\left.\left.\Delta t^{2}\|\boldsymbol{A}\|_{2}+\Delta t^{2}\|\boldsymbol{B}\|_{2}\right)(k-L)\right](k=L+1, L+2, \ldots, K)$ are produced for the extrapolating iteration, which could be used as the suggestions of the POD basis choice and update, respectively. The more concrete expressions are as follows.

(1) In order to ensure the approximate ROESFD solutions with the desirable accuracy, it is necessary to choose $d$ that satisfies

$$
\sqrt{\lambda_{d+1}} \leqslant \max \left\{N^{2-q},(\Delta t)^{2}\right\} .
$$

(2) If $2 \sqrt{\lambda_{d+1}} \exp \left[\left(1+\Delta t^{2}\|\boldsymbol{A}\|_{2}+\Delta t^{2}\|\boldsymbol{B}\|_{2}\right)(k-L)\right]>10 \max \left\{N^{2-q},(\Delta t)^{2}\right\}$ $(L+1 \leqslant k \leqslant K)$, refreshing the POD basis is imperative. If we take $\lambda_{d+1}$ such that $2 \sqrt{\lambda_{d+1}} \exp \left[\left(1+\Delta t^{2}\|\boldsymbol{A}\|_{2}+\Delta t^{2}\|\boldsymbol{B}\|_{2}\right)(k-L)\right]=O\left\{N^{2-q},(\Delta t)^{2}\right\}$ $(L+1 \leqslant k \leqslant K)$, then the ROESFD scheme (3.3) is convergent, hence we don't have to refresh the POD basis.

\subsection{The implementations of the ROESFD scheme}

The following implementation of the ROESFD scheme (3.3) is helpful for understanding the ideas of POD method, which is listed the five steps.

Step 1. For the given $f_{1}, f_{2}$, and $f(x, y, t)$, choose the number of the basis function $N$, take the spatial nodes as $x_{m}=\cos (m \pi / N)(0 \leqslant m \leqslant N)$ and $y_{l}=\cos (l \pi / N)(0 \leqslant l \leqslant N)$, and the time step $\Delta t$ that satisfy the stability condition $\Delta t \leqslant 6.6 N^{-2}$. Write the CSFD scheme as vector form (2.2) and find the first $L$ solution vectors $\boldsymbol{u}^{k}=\left(u_{1}^{k}, u_{2}^{k}, \ldots, u_{n}^{k}, \ldots, u_{M}^{k}\right)^{T}\left(M=(N+1)^{2}\right)$, where $u_{n}^{k}=u_{m, l}^{k}(0 \leqslant m \leqslant N, 0 \leqslant l \leqslant N, 0 \leqslant k \leqslant L)$.

Step 2. Formulate the snapshot matrix $\boldsymbol{P}=\left(\boldsymbol{u}^{1}, \boldsymbol{u}^{2}, \ldots, \boldsymbol{u}^{L}\right)_{M \times L}$ and compute the eigenvalue of matrix $\boldsymbol{P}^{T} \boldsymbol{P}$ according to decreasing order

$$
\lambda_{1} \geqslant \lambda_{2} \geqslant \ldots \geqslant \lambda_{w}>0, \quad w=\operatorname{rank}(\boldsymbol{P})
$$

and the homologous eigenvectors $\boldsymbol{\psi}_{i}(i=1,2, \ldots, w)$.

Step 3. By the error limitation $\sqrt{\lambda_{d+1}} \leqslant \max \left\{N^{2-q}, \Delta t^{2}\right\}$, determine the number of POD basis $d$ and formulate the POD basis $\boldsymbol{\Phi}=\left(\phi_{1}, \phi_{2}, \ldots, \phi_{d}\right)$, where $\boldsymbol{\phi}_{i}=\boldsymbol{P} \boldsymbol{\psi}_{i} / \sqrt{\lambda_{i}}(i=1,2, \ldots, d)$.

Step 4. Solving the ROESFD scheme (3.3) obtains the ROESFD solution vectors $\boldsymbol{u}_{d}^{k}=\left(u_{d 1}^{k}, u_{d 2}^{k}, \ldots, u_{d M}^{k}\right)^{T}(k=1,2, \ldots, L, L+1, \ldots, M)$, further, achieves the components $u_{d, m, l}^{k}=u_{d n}^{k}$.

Step 5. In the previous step, if $2 \sqrt{\lambda_{d+1}} \exp \left[\left(1+\Delta t^{2}\|\boldsymbol{A}\|_{2}+\Delta t^{2}\|\boldsymbol{B}\|_{2}\right)(k-L)\right] \leqslant$ $\max \left\{N^{2-q},(\Delta t)^{2}\right\},(L+1 \leqslant k \leqslant K)$, then, $\boldsymbol{u}_{d}^{k}(k=1,2, \ldots, K)$ are just the ROESFD solution vectors that meet the desirable precision. Else, i.e., if $2 \sqrt{\lambda_{d+1}} \exp \left[\left(1+\Delta t^{2}\|\boldsymbol{A}\|_{2}+\Delta t^{2}\|\boldsymbol{B}\|_{2}\right)(k-L)\right]>10 \max \left\{N^{2-q}, \Delta t^{2}\right\}$, set $\boldsymbol{P}=\left(\boldsymbol{u}_{d}^{z+1}, \boldsymbol{u}_{d}^{z+2}, \ldots, \boldsymbol{u}_{d}^{z+L}\right)(z=k-1-L)$ as the new snapshot matrix, return to Step 2. 


\section{Some numerical examples}

Here, we supply three familiar numerical examples to validate the feasibility and availability of the ROESFD algorithm based on the POD method for the $2 \mathrm{D}$ second-order hyperbolic equations (1.1).

Example 1. In the hyperbolic equations (1.1), we took $f(x, y, t)=0, f_{1}(x)=$ $\sin \pi x \sin \pi y, f_{2}(x)=0$, the number of the basis functions $N=90$. And, the time step was taken as $\Delta t=0.0005$, which satisfied the stability condition $\Delta t \leqslant 6.6 N^{-2}$.

Using the CSFD scheme (2.2), we got the CSFD solution at $T=0.2$, which was depicted graphically in Figure 1(a). We took the first 20 CSFD solutions as snapshots. According to the Step 2 and Step 3 in Section 3.4, if we use the first six eigenvectors of the matrix $\boldsymbol{P} \boldsymbol{P}^{T}$ as the POD basis, the theoretical precision requirement can be satisfied. Finally, we computed the ROESFD solution at $T=0.2$ by mean of the ROESFD scheme (3.3) with six POD bases, which was depicted graphically in Figure 1(b).

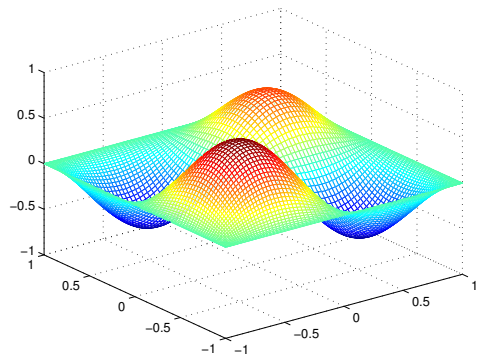

(a)

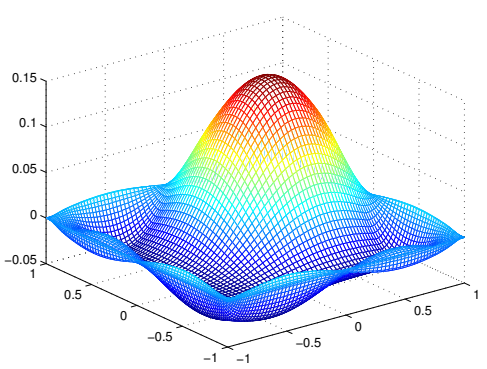

(c)

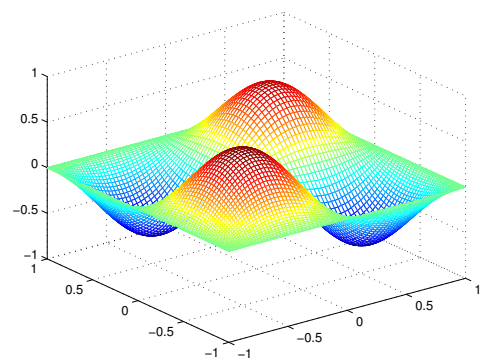

(b)

Figure 1. The solutions at $T=0.2$ : (a) CSFD solution, (b) ROESFD solution, (c) the relative error chart.

Figure 1 (c) shows the relative error chart between the CSFD solution and the ROESFD solution at $T=0.2$, which is accordant with theoretical result. Compared the CSFD scheme, we could clearly realize the advantages of the ROESDF scheme based on the POD technique. 
First, the CSFD solution graph (Figure 1(a)) and the ROESFD solution (Figure 1(b)) were basically identical. Because the ROESFD scheme (3.3) based on the POD technique only included six unknowns whereas the CSFD scheme (2.2) contained 10000 degrees of freedom, the ROESFD scheme can significantly lessen the degrees of freedom so that moderate the accumulation of the truncated error. Therefore, we could clearly see that the ROESFD solution was more smooth and steady than the CSFD solution. Second, the ROESFD scheme based on the POD technique could save more computing time than the CSFD scheme because the operation time of the CSFD scheme is $48.07 \mathrm{~s}$ whereas that of the ROESFD scheme is $4.11 \mathrm{~s}$ in the same computer.

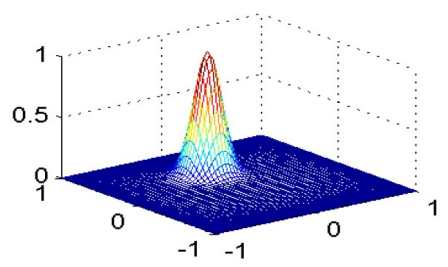

(a) CSFD solutions at $\mathrm{T}=0$

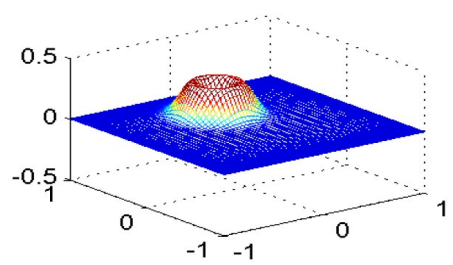

(c) CSFD solutions at $\mathrm{T}=0.15$

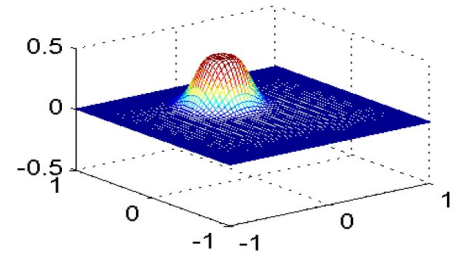

(b) CSFD solutions at $\mathrm{T}=0.1$

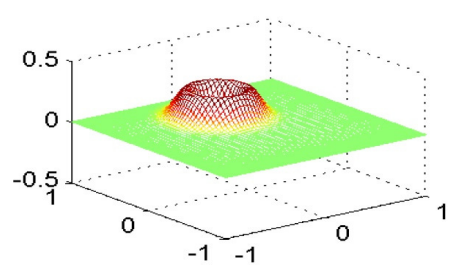

(d) CSFD solutions at $\mathrm{T}=0.2$

Figure 2. The CSFD solutions at $T=0,0.1,0.15,0.2$.

Example 2. In the hyperbolic equations (1.1), we took $f(x, y, t)=0, f_{1}(x)=$ $e^{-40\left((x-0.4)^{2}+y^{2}\right)}, f_{2}(x)=0$, the number of the basis function $N=90$. And the time step was laso taken as $\Delta t=0.0005$ in order to satisfy the stability condition $\Delta t \leqslant 6.6 N^{-2}$.

Using the CSFD scheme (2.2), we got the CSFD solutions at $T=0,0.1$, $0.15,0.2$, which were depicted graphically in Figure 2. We took the CSFD solution in the first 20 steps as snapshots. According to the Step 2 and Step 3 in Section 3.4, we achieved $\sqrt{\lambda_{6}} \leqslant 25 \times 10^{-8}$, which showed that as long as we took the first five eigenvectors of matrix $\boldsymbol{P} \boldsymbol{P}^{T}$ as the POD basis, the theoretical precision could be satisfied. Finally, we computed the ROESFD solutions at $T=0,0.1,0.15,0.2$ by mean of the ROESFD scheme (3.3) with ten POD bases, which were depicted graphically in Figure 3.

From the charts above, we could also obtain the same conclusions as the Example 1 that the CSFD solution graph (Figure 2) and the ROESFD solution one (Figure 3) were basically identical and the ROESFD solution chart 


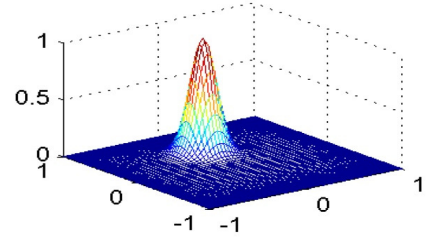

(a) ROESFD solutions at $\mathrm{T}=0$

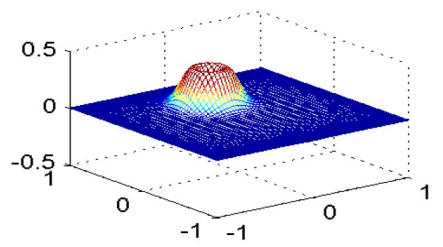

(c) ROESFD solutions at $\mathrm{T}=0.15$

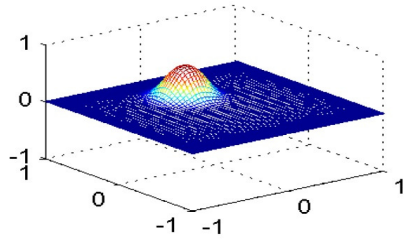

(b) ROESFD solutions at $\mathrm{T}=0.1$

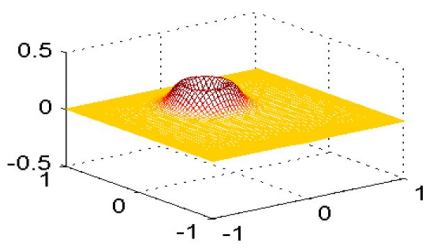

(d) ROESFD solutions at $\mathrm{T}=0.2$

Figure 3. The ROESFD solutions at $T=0,0.1,0.15,0.2$.

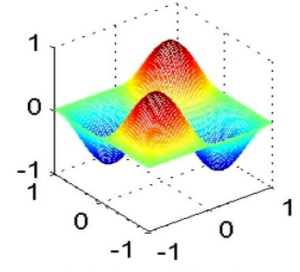

(a) Exact solution at $\mathrm{T}=0.2$

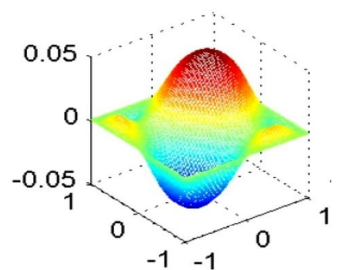

(d) Error between exact and CSFD solutions

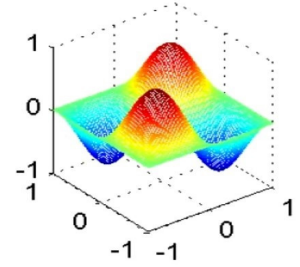

(b) CSFD solution at $\mathrm{T}=0.2$

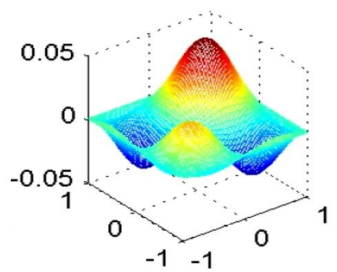

(e) Error between exact and ROESFD solutions

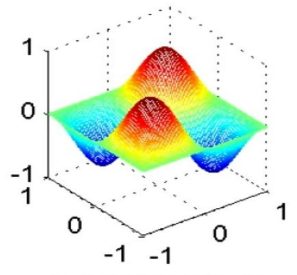

(c) ROESFD solution at $\mathrm{T}=0.2$

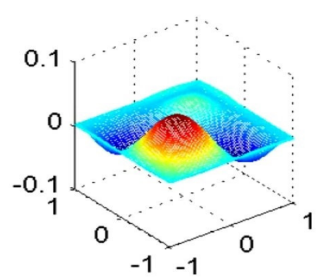

(f) Error between CSFD and ROESFD solutions

Figure 4. The exact, CSFD, ROESFD solution at $T=0.2$ in the first line, the error between exact and CSFD solution, exact and ROESFD solution, CSFD and ROESFD solution at $T=0.2$.

was more smooth and steady compared with the CSFD solution one. By computation, we found that the max relative error of the solutions for two different spectral-FE schemes at $T=0.2$ does not exceed $0.0286 \times 10^{-4}$. Moreover, the ROESFD scheme could save more computing time than the CSFD one because the operation time of the CSFD scheme, also including 10000 unknowns, is $50.59 \mathrm{~s}$ at the MATLAB software, whereas that of the ROESFD scheme is 
$5.14 s$ in the same computer.

Example 3. In the hyperbolic equations (1.1), we took $f(x, y, t)=0, f_{1}(x)=$ $0, f_{2}(x)=\sqrt{2} \pi \sin \pi x \sin \pi y$. Thus, the analytical solution of the equation was $u(x, y, t)=\sin (\sqrt{2} \pi t) \sin \pi x \sin \pi y$. We still took the number of the basis function $N=99$ and the time step was also taken as $\Delta t=0.0005 .1$

Using the same approaches as the examples above, the images of the exact solution, the CSFD solution, and the ROESFD solution at $T=0.2$ were, respectively, depicted in the Charts (a), (b), and (c) in Figure 4. In order to illustrate the correctness of the CSFD method and the ROESFD method, we provided the error images between the exact solution and the CSFD one, the exact solution and the ROESFD one, and the CSFD solution and the ROESFD one in Charts (d), (e), and (f) in Figure 4, respectively.

From the Figure 4, we could see that the exact solution graph, the CSFD solution graph, and the ROESFD solution one were basically identical and its error is extreme small. Moreover, the ROESFD scheme could save more computing time than the CSFD one.

\section{Conclusions and discussion}

In this study, we have set up the CSFD scheme and the ROESFD scheme based on the POD technique for the 2D second-order hyperbolic equations, analyzed the errors and stability conditions of these two algorithms, and provided the implementation for the ROESFD scheme. The numerical examples have confirmed the correctness of our theoretical analysis and illustrated that the ROESFD scheme based on the POD technique has advantages with lessening calculation load, saving computing time, alleviating the truncation error accumulation, and improving computation efficiency compared with the CSFD scheme when deals with the $2 \mathrm{D}$ second-order hyperbolic equations.

Although we restrict our scheme for the $2 \mathrm{D}$ second-order hyperbolic equations on the domain $\bar{\Omega}=[a, b] \times[c, d]$, our technique can extend to the bigger or more general domains, even extend to the more complicated engineering problems. Therefore, our technique has important applied prospect.

\section{Acknowledgements}

Author would like to acknowledge cordially four anonymous referees and editors for their valuable comments which lead to the improvement of this paper.

\section{References}

[1] J. An, Z. Luo, H. Li and P. Sun. Reduced-order extrapolation spectralfinite difference scheme based on POD method and error estimation for threedimensional parabolic equation. Front. Math. China, 10(5):1025-1040, 2015. https://doi.org/10.1007/s11464-015-0469-8.

[2] N. Aubry, P. Holmes, J.L. Lumley and E. Stone. The dynamics of coherent structures in the wall region of a turbulent boundary layer. J. Fluid Dyn., 192:115-173, 1988. https://doi.org/10.1017/s0022112088001818. 
[3] W. Cazemier, R.W.C.P. Verstappen and A.E.P. Veldman. Proper orthogonal decomposition and low-dimensinal models for driven cavity flows. Physics of Fluids, 10(7):1685-1699, 1998. https://doi.org/10.1063/1.869686.

[4] K. Fukunaga. Introduction to Statistical Recognition. Academic Press, New York, 1990.

[5] B.Y. Guo. Spectral methods and their applications. Singapore, World Scientific, 1998. https://doi.org/10.1142/3662.

[6] P. Holmes, J.L. Lumley and G. Berkooz. Turbulence, Coherent Structures, Dynamical Systems and Symmetry. Cambridge University Press, Cambridge, 1996. https://doi.org/10.1017/CBO9780511622700.

[7] G. Jankevičiūtè, T. Leonavičienè and R. Čiegis. Reduced order modelling based on POD method for Schrödinger equation. Math. Model. Anal., 18(5):694-707, 2013. https://doi.org/10.3846/13926292.2013.870611.

[8] K. Kunisch and S. Volkwein. Galerkin proper orthogonal decomposition methods for parabolic problems. Numer. Math., 90(1):117-148, 2001. https://doi.org/10.1007/s002110100282.

[9] K. Kunisch and S. Volkwein. Galerkin proper orthogonal decomposition methods for a general equation in fluid dynamics. SIMA J. Numer. Anal., 40(2):492-515, 2002. https://doi.org/10.1137/S0036142900382612.

[10] Z. Liu. Collocation-Spectral for Three-Dimensional Parabolic Equations. Heilongjiang University, Harbin, 2015.

[11] Z. Luo. A POD-based reduced-order stabilized Crank-Nicolson MFE formulation for the no-stationary parabolized Navier-Stokes equations. Math. Model. Anal., 20(3):346-368, 2015. https://doi.org/10.3846/13926292.2015.1048758.

[12] Z. Luo. A POD-based reduced-order TSCFE extrapolation iterative format for two-dimensional heat equations. Bound. Value Probl., 2015(1):1-15, 2015. https://doi.org/10.1186/s13661-015-0320-x.

[13] Z. Luo. Proper orthogonal decomposition-based reduced-order stabilized mixed finite volume element extrapolating model for the non-stationary incompressible Boussinesq equations. J. Math. Anal. Appl., 425(1):259-280, 2015. https://doi.org/10.1016/j.jmaa.2014.12.011.

[14] Z. Luo, J. Du, Z. Xie and Y. Guo. A reduced stabilized mixed finite element formulation based on proper orthogonal decomposition for the nostationary Navier-Stokes equations. J. Numer. Meth. Eng., 88(1):31-46, 2011. https://doi.org/10.1002/nme.3161.

[15] Z. Luo, S. Jin and J. Chen. A reduced-order extrapolation central difference scheme based on POD for two dimensional fourthorder hyperbolic equations. Appl. Math. Comput., 289:396-408, 2016. https://doi.org/10.1016/j.amc.2016.05.032.

[16] Z. Luo, H. Li, J. Chen and F. Teng. A reduced-order FVE extrapolation algorithm based on proper orthogonal decomposition technique and its error analysis for Sobolev equation. Japan J. Indust. Appl. Math., 32(1):119-142, 2015. https://doi.org/10.1007/s13160-014-0162-4.

[17] Z. Luo, H. Li, P. Sun and J. Gao. A reduced-order finite difference extrapolation algorithm based on POD technique for the non-stationary Navier-Stokes equations. Appl. Math. Model., 37(7):5464-5473, 2013. https://doi.org/10.1016/j.apm.2012.10.051. 
[18] Z. Luo, Q. Ou, J. Wu and Z. Xie. A reduced FE formulation based on POD method for hyperbolic equation. Acta Math. Sci., 32(5):1997-2009, 2012. https://doi.org/10.1016/S0252-9602(12)60155-6.

[19] Z. Luo, D. Xie and F. Teng. A POD-based reduced-order FD extrapolating algorithm for traffic flow. Adv. Difference Equ., 2014(269):1-13, 2014. https://doi.org/10.1186/1687-1847-2014-269.

[20] Z. Luo, Z. Xie, Y. Shang and J. Chen. A reduced finite volume element formulation based on POD for parabolic equations. J. Comput. Appl. Math., 235(8):2098-2111, 2011. https://doi.org/10.1016/j.cam.2010.10.008.

[21] Z. Luo, X. Yang and Y. Zhou. A reduced finite difference scheme based on singular value decomposition and proper orthogonal decomposition for Burgers equation. J. Comput. Appl. Math., 229(1):97-107, 2009. https://doi.org/10.1016/j.cam.2008.10.026.

[22] H.V. Ly and H.T. Tran. Proper orthogonal decomposition for flow calculations and optimal control in a horizontal CVD reactor. J. Comput. Appl. Math., 60(4):631-656, 2002. https://doi.org/10.1090/qam/1939004.

[23] P. Moin and R.D. Moser. Characteristic-eddy decomposition of turbulence in a channel. J. Fluid Mech., 200:471-509, 1989. https://doi.org/10.1017/S0022112089000741.

[24] J.D. Ren. High-order compact difference method for solving two dimensional wave equation. Appl. Research Comput., 29(6):2112-2116, 2012.

[25] J. Shen and T. Tang. Spectral and High-Order Methods with Applications. Science Press, Beijing, 2006.

[26] L. Sirovich. Turbulence and the dynamics of coherent structures. I Coherent structures. II Symmetries and transformations. III Dynamics and scaling. Quart. Appl. Math., 45(3):561-571, 1987. https://doi.org/10.1090/qam/910462.

[27] P. Sun, Z. Luo and Y. Zhou. Some reduced finite difference schemes based on a proper orthogonal decomposition technique for parabolic equations. Appl. Numer. Math., $\mathbf{6 0}(1): 154-164,2010$. https://doi.org/10.1016/j.apnum.2009.10.008.

[28] F. Teng, Z. Luo and X. Li. A POD-based reduced-order finite difference extrapolation iterative format for 2D hyperbolic equation. Appl. Math. J. Chinese Univ., 29(4):389-396, 2014.

[29] L.N. Trefrthen. Spectral Methods in MATLAB. SIAM, Beijing, 2000. https://doi.org/10.1137/1.9780898719598.

[30] W.S. Zhang. Finite Difference Methods for Patial Differential Equations in Science Computation. Higher Education Press, Beijing, 2006. 